\title{
Extended State Observer (ESO) Based Control Strategy with Parameter Optimization for Brushless Doubly-fed Machine
}

\author{
Hai-yu Guo \\ School of Electrical Engineering \\ Shenyang University of Technology \\ Shenyang, China \\ ghy@sut.edu.cn
}

\author{
Xiao-guang Zhang \\ Brilliance Automotive Engineering Research Institute \\ Shenyang, China \\ zhangxiaoguang@brilliance-auto.com
}

\begin{abstract}
This work is concerned with an extended state observer (ESO) based controller with parameter optimization for brushless doubly-fed machine (BDFM). An ESO based controller is proposed to deal with the complex inner coupling effect and external disturbance, which are hardly obtained in the practice. To reduce the workload of the parameters selection, an optimized parameter design method for the nonlinear ESO is proposed. Simulation results show that the proposed controller, which brings out an excellent performance and increases the robustness of the system, rejects the disturbances successfully and achieves the decoupling control of the system.
\end{abstract}

Keywords-Brushless doubly-fed machine; extended state observer; disturbance rejection; parameter optimization

\section{INTRODUCTION}

The Brushless Doubly-Fed Machine (BDFM) retains the Doubly-Fed Induction Machine (DFIM) cost benefit of utilizing a small converter, but provides higher reliability by removing brush gear and slip ring, showing significant commercial benefits for use both in variable speed drives and wind power generation.

BDFM is a complex nonlinear system with multiple variables and strong coupling effects. To date, the control strategies have been proposed for BDFM mainly involve direct torque control (DTC) and vector control. DTC for BDFM causes fluctuations in torque and current. There have been some works ${ }^{[1]}$ focused on improvements. But new research is still in need on the excellent DTC strategy, which can eliminate the ripples completely and maintain the advantage of low dependence on parameters and model. The vector control for BDFM ${ }^{[2-3]}$ strongly depends on the model and parameters of the object. A simple, high performance decoupling control method is in urgent need. For complex nonlinear systems, the nonlinear control strategies ${ }^{[4]}$ show more and more obvious advantages in recent years. This paper proposes an extended state observer (ESO) ${ }^{[5]}$ based control strategy to achieve the simple but high performance control for BDFM. By estimating and compensating the disturbance and uncertainties via an ESO in real time, the complex nonlinear system is transformed into an integral serial linear system. Then the design of the control law is simpler. This controller depends neither on an accurate model nor the

\footnotetext{
Sponsored by:

1. China national energy application technology research and

engineering demonstration project (NY20150303);

2. Liaoning provincial department of education science and technology

research project (201634091)
}

parameters of the plant, and has a greater disturbance rejection capability and more robustness to the uncertainties than the conventional PID controller. To simplify the parameter tuning, a parameter configuration method based on the concept of bandwidth for the linear ESO (LESO) is proposed in reference [6]. However, a nonlinear ESO with appropriate parameters provides a better performance than a LESO. To date, the ESO has been widely used in the practical engineering ${ }^{[7-9]}$. In this paper, the nonlinear ESO with parameter optimization is utilized to provide an excellent performance but with less tuning work.

Fig. 1 shows a cascade BDFM. It is a coaxial series of two wound induction motors whose rotors windings are connected in an opposite phase. The stator of one motor, called the power motor (PM), is often connected to the grid. The stator of the other motor, called the control motor (CM), is supplied through a converter. By adjusting the amplitude and frequency of the CM stator voltage, the CM flux and electromagnetic torque of BDFM can be controlled successfully. This machine is referred to simply as BDFM for the sake of convenience, from here on.

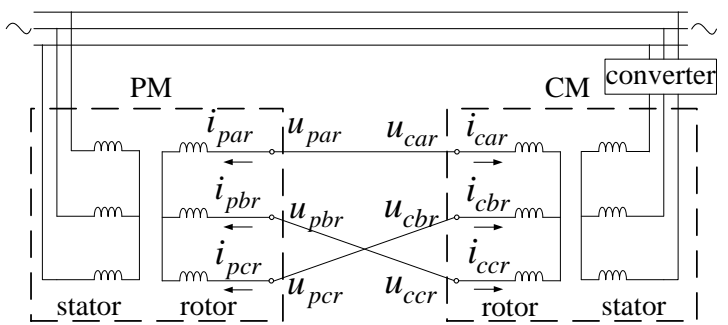

Fig. 1. Structure of cascade wound-rotor BDFM

TABLE I. EXPLANATION OF SYMBOLS TABLE STYLES

\begin{tabular}{|c|c|c|c|}
\hline symbol & explanation & symbol & explanation \\
\hline$r$ & resistance & $T_{e}$ & electromagnetic torque \\
\hline$l$ & inductance & $f$ & supply frequency \\
\hline$l_{m}$ & mutual inductance & $w_{r}$ & rotor's angular speed \\
\hline$i$ & current & $J$ & moment of inertia \\
\hline$u$ & voltage & $p$ & pole pairs number \\
\hline$\psi$ & flux & Subscripts & explanation \\
\hline
\end{tabular}




\begin{tabular}{|c|c|c|c|}
\hline \multicolumn{4}{|c|}{ Cont. to table I } \\
\hline$n_{r}$ & motor speed & $s, r$ & stator (rotor) winding \\
\hline$T_{l}$ & load torque & $p, c$ & power (control) motor \\
\hline
\end{tabular}

In this paper, the explanation of symbols in this paper is given in Table 1.

\section{Mathematical Model in SynChronous ReferenCE FRAME OF BDFM}

In this paper, $\boldsymbol{x}=\operatorname{Re} \boldsymbol{x}+j \operatorname{Im} \boldsymbol{x}$ represents a complex vector. The fluxes of PM and CM are

$$
\left\{\begin{array}{l}
\boldsymbol{\psi}_{p s}^{\mathrm{dq}}=l_{p s} \mathbf{i}_{p s}^{\mathrm{dq}}+l_{p m} \mathbf{i}_{p r}^{\mathrm{dq}} \\
\boldsymbol{\psi}_{p r}^{\mathrm{dq}}=l_{p m} \boldsymbol{i}_{p s}^{\mathrm{dq}}+l_{p r} i_{p r}^{\mathrm{dq}} \\
\boldsymbol{\psi}_{c s}^{\mathrm{dq}}=l_{c s} \mathbf{i}_{c s}^{\mathrm{dq}}+l_{c m} \mathbf{i}_{c r}^{\mathrm{dq}} \\
\boldsymbol{\psi}_{c r}^{\mathrm{dq}}=l_{c m} \boldsymbol{i}_{c s}^{\mathrm{dq}}+l_{c r} \mathbf{i}_{c r}^{\mathrm{dq}}
\end{array}\right.
$$

The electromagnetic torques of them is:

$$
\left\{\begin{array}{l}
T_{e p}=p_{p} l_{p r} \operatorname{Im}\left(i_{p r}^{* \mathrm{dq}} i_{p s}^{\mathrm{dq}}\right) \\
T_{e c}=p_{c} l_{c r} \operatorname{Im}\left(i_{c r}^{* \mathrm{dq}} \mathbf{i}_{c s}^{\mathrm{dq}}\right)
\end{array}\right.
$$

Where ' $*$ ' is the conjugate operator. The two rotor windings of BDFM are connected in an opposite phase, as shown in Fig.1, so the relationship between the phase voltages and line currents of the two rotors are as follows:

$$
\left\{\begin{array}{l}
u_{p a r}=u_{c a r} \\
u_{p b r}=u_{c c r} \\
u_{p c r}=u_{c b r}
\end{array},\left\{\begin{array}{l}
i_{p a r}=-i_{c a r} \\
i_{p b r}=-i_{c c r} \\
i_{p c r}=-i_{c b r}
\end{array}\right.\right.
$$

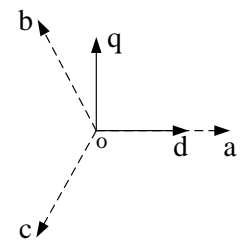

Fig. 2. Rotor dq reference frame and rotor abc reference frame

Then, we have:

$$
\boldsymbol{u}_{p r}^{\mathrm{dq}}=\boldsymbol{u}_{c r}^{* \mathrm{dq}}, \mathbf{i}_{p r}^{\mathrm{dq}}=-\mathbf{i}_{c r}^{* \mathrm{dq}}
$$

To unify the equations of the PM and the CM, We represent $\boldsymbol{i}_{p r}$ and $\boldsymbol{i}_{c r}$ by $\boldsymbol{i}_{r}$, and set $\boldsymbol{i}_{r}=\boldsymbol{i}_{c r}$, i.e.,

$$
\boldsymbol{i}_{p r}^{\mathrm{dq}}=-\boldsymbol{i}_{c r}^{* \mathrm{dq}}=-\boldsymbol{i}_{r}^{* \mathrm{dq}}
$$

Then, the BDFM model in dq frame is

$$
\left\{\begin{array}{l}
\boldsymbol{u}_{p s}^{* \mathrm{dq}}=r_{p s} \boldsymbol{i}_{p s}^{* \mathrm{dq}}+\frac{d \boldsymbol{\psi}_{p s}^{* \mathrm{dq}}}{d t}-j p_{p} \omega_{r} \boldsymbol{\psi}_{p s}^{* \mathrm{dq}} \\
\boldsymbol{u}_{c s}^{\mathrm{dq}}=r_{c s} \mathbf{i}_{c s}^{\mathrm{dq}}+\frac{d \boldsymbol{\psi}_{c s}^{\mathrm{dq}}}{d t}+j p_{c} \omega_{r} \boldsymbol{\psi}_{c s}^{\mathrm{dq}} \\
0=r_{r} \boldsymbol{i}_{r}^{\mathrm{dq}}+\frac{d \boldsymbol{\psi}_{c r}^{\mathrm{dq}}}{d t}-\frac{d \boldsymbol{\psi}_{p r}^{* \mathrm{dq}}}{d t}
\end{array}\right.
$$

Where $r_{r}=r_{p r}+r_{c r}$. The motion equation of BDFM is:

$$
J \frac{d \omega_{r}}{d t}=T_{e}-T_{L}
$$

Obviously, the variables are AC quantities rather than DC values in the rotor $\mathrm{dq}$ frame. To facilitate control, we transform the variables into the control motor synchronous reference frame. Given an two-axis mt reference frame, and we orient this reference frame on the CM rotor flux, to produce:

$$
\psi_{c r}^{\mathrm{mt}}=\psi_{c m r}+j \psi_{c t r}=\psi_{c r}+j 0
$$

where $\psi_{c r}$ is the amplitude of the CM rotor flux. And the angle between $\mathrm{mt}$ reference frame and dq reference frame is $\lambda_{c}$, as shown in Fig. 3.

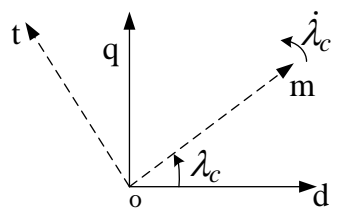

Fig. 3. rotor dq reference frame, and synchronous mt reference frame

Expressions of the flux and speed in the mt reference frame are obtained by coordinate transformation:

$$
\begin{aligned}
\dot{\psi}_{c r}= & \frac{l_{c r} l_{p m} r_{p s}}{l_{p s}\left(l_{r} l_{p s}-l_{p m}^{2}\right)} \psi_{p m s}^{\prime}+\frac{l_{c r} l_{p m}}{\left(l_{r} l_{p s}-l_{p m}^{2}\right)} p_{p} \omega_{r} \psi_{p t s}^{\prime} \\
& -\frac{\left(l_{p m}^{2} r_{p s}+l_{p s}^{2} r_{r}\right)}{l_{p s}\left(l_{r} l_{p s}-l_{p m}^{2}\right)} \psi_{c r}-\frac{l_{c r} l_{p m}}{\left(l_{r} l_{p s}-l_{p m}^{2}\right)} u_{p m s}^{\prime} \\
& +\frac{l_{c m}\left(l_{p m}^{2} r_{p s}+l_{p s}^{2} r_{r}\right)}{l_{p s}\left(l_{r} l_{p s}-l_{p m}^{2}\right)} i_{c m s}+\frac{l_{c m}\left(l_{p r} l_{p s}-l_{p m}^{2}\right)}{\left(l_{r} l_{p s}-l_{p m}^{2}\right)} \dot{c}_{c m s} \\
& T_{e}=p_{p}\left(\psi_{p t s}^{\prime} i_{p m s}^{\prime}-\psi_{p m s}^{\prime} i_{p t s}^{\prime}\right)+\frac{p_{c} l_{c m}}{l_{c r}} \psi_{c r} i_{c t s}
\end{aligned}
$$

Where $l_{r}=l_{p r}+l_{c r}$

In actuality, the control motor is generally designed with an inner current loop. Because of the sufficiently rapid response of the current loop, the machine is considered to be supplied by a current source. The flux and the speed can be controlled by the excitation current $i_{c m s}$ and the torque current $i_{c t s}$, respectively.

\section{ESO BASED CONTROLLER FOR BDFM}

As shown in (8)-(9), the flux is coupled with the torque strongly. We treat this coupling effect as the inner disturbance of the system, and the unknown load as the external disturbance. ESOs are utilized to estimate and eliminate these disturbances.

\section{A. ESO based controller for the CM rotor flux}

The CM rotor flux equation (8) can be expressed as: 


$$
\dot{\psi}_{c r}=a_{1}(x)+b_{1} u
$$

Where $u=i_{\text {cms }}, a_{1}(x)$ represents the uncertainties, $b_{1}$ is an unknown parameter. We transform (10) into a standard form as:

$$
\left\{\begin{array}{l}
\dot{\psi}_{c r}=f+b_{10} u \\
y=\psi_{c r}
\end{array}\right.
$$

Where $f$ represents the total disturbance in the flux loop, and $f=a_{1}(x)+\left(b_{1}-b_{10}\right) u$. According to (11), we design a second-order ESO for the flux as

$$
\left\{\begin{array}{l}
e=z_{1}-y \\
\dot{z}_{1}=z_{2}-\beta_{1} \mathrm{fal}\left(e, \alpha_{1}, \delta_{1}\right)+b_{10} u \\
\dot{z}_{2}=-\beta_{2} \mathrm{fal}\left(e, \alpha_{1} / 2, \delta_{1}\right)
\end{array}\right.
$$

where $y$ is the output $\psi_{c r}, z_{1}$ is the estimation of $y$, $z_{2}$ is the estimation of the total disturbance $f, e$ is the error between output and its estimation, $\beta_{1}$ and $\beta_{2}$ are the parameters of the second-order ESO, which are usually adjusted according to the control performance. The nonlinear function has the form as

$$
\operatorname{fal}(e, \alpha, \delta)=\left\{\begin{array}{l}
\frac{e}{\delta^{1-\alpha}}, \quad|e| \leq \delta \\
\operatorname{sgn}(e)|e|^{\alpha}, \quad|e|>\delta
\end{array}\right.
$$

where $\operatorname{sgn}(\cdot)$ is the sign function. The fal function adjusts the control gain according to the error. When $\alpha<1$, the gain is large as the error is small, while it is small when the error is large.

Set a new input $u_{0}$, and let

$$
u=\frac{u_{0}-z_{2}}{b_{10}}
$$

Then the system is equal to the linear system as

$$
\dot{\psi}_{c r}=u_{0}
$$

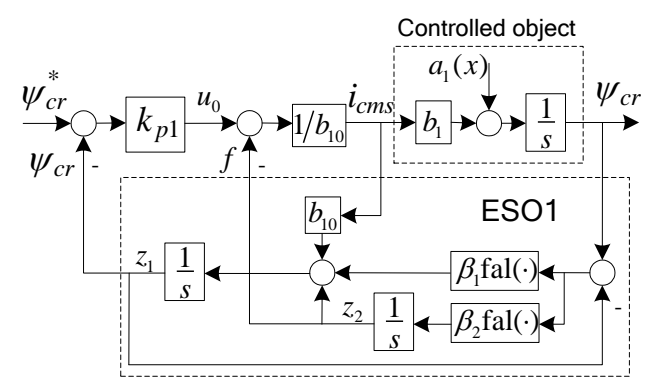

Fig. 4. ESO based controller for the CM flux

The structure of the controller is shown in Fig.4.For the linear system as Eq. (14), as the disturbances are estimated and eliminated via the ESO, there is no need for integral control. It is effective to choose the simple proportional control as the control law. So the final flux controller is

$$
i_{c m s}=\frac{u_{0}-z_{2}}{b_{10}}=\frac{k_{p 1}\left(\psi_{c r}^{*}-\psi_{c r}\right)-z_{2}}{b_{10}}
$$

\section{B. ESO based controller for the motor speed}

According to Eqs. (6) and (9), the motor speed can be expressed as:

$$
\dot{\omega}_{r}=a_{2}(x)+b_{2} u
$$

Where $u=i_{c t s}, b_{2}=p_{c} l_{c m} \psi_{c r} /\left(J l_{c r}^{\prime}\right)$ is usually an unknown parameter in practice, $a_{2}(x)$ represents the uncertainties. We rewrite (16) in the standardized form:

$$
\left\{\begin{array}{l}
\dot{\omega}_{r}=f+b_{20} u \\
y=\omega_{r}
\end{array}\right.
$$

Where $f=a_{2}(x)+\left(b_{2}-b_{20}\right) u$. Similarly, a second-order ESO is used for the rotor speed, as

$$
\left\{\begin{array}{l}
e=z_{1}-y \\
\dot{z}_{1}=z_{2}-\beta_{3} \operatorname{fal}\left(e, \alpha_{2}, \delta_{2}\right)+b_{20} u \\
\dot{z}_{2}=-\beta_{4} \operatorname{fal}\left(e, \alpha_{2} / 2, \delta_{2}\right)
\end{array}\right.
$$

Set a new input $u_{0}$, and the final speed controller is

$$
u=\frac{u_{0}-z_{2}}{b_{20}}=\frac{k_{p 2}\left(\omega_{r}^{*}-\omega_{r}\right)-z_{2}}{b_{20}}
$$

\section{Parameter optimization for the ESO}

Parameter configuration is a difficulty for the design of a nonlinear ESO, but not for the LESO. The parameters of a LESO can be selected according to the LESO's closed-loop transfer function ${ }^{[6]}$. So we select the nonlinear ESO's parameters based on the above rule of LESO. By appropriate adjustment, a nonlinear ESO with a better performance by less work on parameter configuration is obtained.

Second-order ESOs are utilized in this paper. According to the parameter selecting method in reference [6], given a second-order LESO designed as

$$
\left\{\begin{array}{l}
e=z_{1}-y \\
\dot{z}_{1}=z_{2}-\beta_{1}\left(z_{1}-y\right)+b_{10} u \\
\dot{z}_{2}=-\beta_{2}\left(z_{1}-y\right)
\end{array}\right.
$$

the parameters are designed as

$$
\beta_{1}=2 \omega_{o}, \quad \beta_{2}=\omega_{o}^{2}
$$

where $\omega_{o}$ is the observer bandwidth. And the LESO's response time is inversely proportional to $\omega_{0}$. The flux subsystem (8) is linearized via the LESO, and according to (14)-(15), the closed loop transfer function of this subsystem is 


$$
G_{c 1}(s)=\frac{k_{p 1}}{s+k_{p 1}}
$$

Similarly, the proportional controller's parameter is selected as

$$
k_{p 1}=\omega_{c}
$$

where $\omega_{c}$ is the controller bandwidth. As the parameters $\beta_{1}$, $\beta_{2}$ and $k_{p 1}$ of the LESO can be designed by (21) and (23), we also design the parameters $\beta_{1}, \beta_{2}$ and $k_{p 1}\left(\beta_{3}\right.$, $\beta_{4}$ and $k_{p 2}$ ) of the nonlinear ESO by the same rules, while adjust the rest parameters $\alpha_{1}$ and $\delta_{1}\left(\alpha_{2}\right.$ and $\left.\delta_{2}\right)$ to get a better performance. Fig. 5 displays the performance comparison between the LESO and the nonlinear ESO with different $\alpha$ and $\delta$.

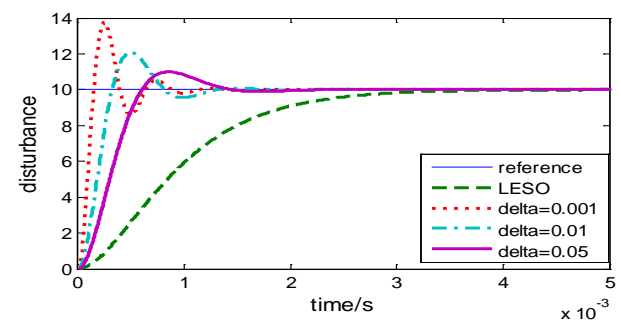

(a) $\omega_{0}=2000, \alpha=0.9, \quad \delta$ (delta) varies.

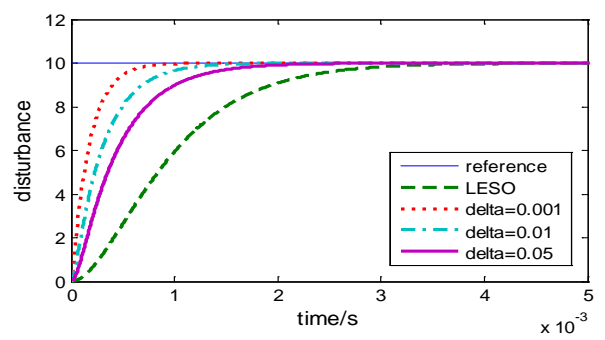

(b) $\omega_{0}=2000, \alpha=0.5, \delta$ (delta) varies.



(e) $\omega_{o}=200, \delta=0.01, \alpha$ (alfa) varies.

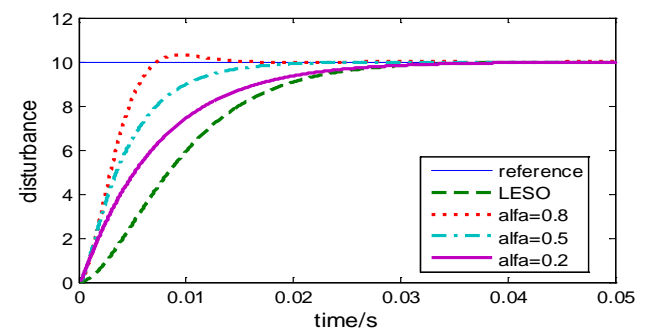

(f) $\omega_{0}=200, \delta=0.05, \alpha$ (alfa) varies.

Fig. 5. observers' performances comparison between ESO and LESO
As shown in Fig.5, both $\alpha$ and $\delta$ affect the ESO's performance. A bigger $\alpha$ causes a quicker response. However, overshoots appear when $\alpha$ is over big ( $\alpha=0.8$ ). While the smaller $\delta$ is, the faster the ESO responses. But a too small $\delta$ will cause a high frequency flutter in a practical system. To obtain a performance better enough than the LESO, we choose $\alpha_{1}=\alpha_{2}=0.5$ and $\delta_{1}=\delta_{2}=0.01$ for the nonlinear ESOs in this paper.

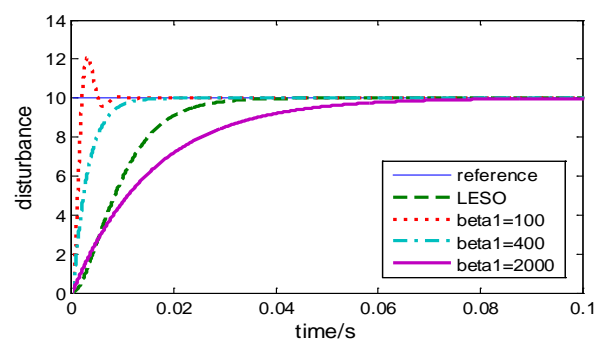

Fig. 6. observers' performances comparison between ESO and LESO ( $E S O: \alpha=0.5, \delta=0.01, \beta_{1}$ (beta1) varies, $\beta_{2}=200 * 200=40000$; LESO : $\omega_{o}=200$.)

Besides, to illustrate the parameters configuration rule in (21) is effective and preferable to design the parameters $\beta_{1}$ and $\beta_{2}$ for the nonlinear ESO (12), we make a comparison between the ESOs with the same $\beta_{2}$ but different $\beta_{1}$ in Fig. 6. As displayed in Fig.6, when the parameters accord with the configuration rule in (21) $\left(\beta_{1}=2 \omega_{o}=400\right)$, the ESO provides an excellent performance. But, overshoots appear if we select a smaller $\beta_{1} \quad\left(=0.5 \omega_{0}=100\right)$. Furthermore, if we set $\beta_{1}$ too big $\left(\beta_{1}=10 \omega_{o}=2000\right)$, the ESO even responses slower than the LESO. Therefore, it is sensible to design $\beta_{1}$ and $\beta_{2}$ for the nonlinear ESO by the rule in (21).

\section{SimUlation RESUltS AND ANALYSIS}

\section{A. Simulation setup}

In the following simulations, the PM $(380 \mathrm{~V} / 50 \mathrm{~Hz} /$ $9.8 \mathrm{~A} / 3.7 \mathrm{KW}$ ) is connected to a $220 \mathrm{~V} / 50 \mathrm{~Hz}$ power supply, and the $\mathrm{CM}(380 \mathrm{~V} / 50 \mathrm{~Hz} / 9.2 \mathrm{~A} / 3.7 \mathrm{KW})$ is supplied through an inverter. The switching frequency of SPWM is $10 \mathrm{kHz}$, and the DC voltage of the inverter is $300 \mathrm{~V}$. Measurement filters are considered for the voltage $(1 \mathrm{~ms})$, current $(0.1 \mathrm{~ms})$, and rotor speed (1ms). The other parameters of BDFM are as follows: $p_{p}=1, r_{p s}=1.77 \Omega, r_{p r}=2.97 \Omega, l_{p s}=l_{p r}=0.461 H$, $l_{p m}=0.458 \mathrm{H}, \quad J=0.1 \mathrm{~kg} \cdot \mathrm{m}^{2}, \quad p_{c}=3, r_{c s}=1.71 \Omega$, $r_{c r}=3.03 \Omega, l_{c s}=l_{c r}=0.13 \mathrm{H}, l_{c m}=0.125 \mathrm{H}, \mathrm{J}=0.1 \mathrm{~kg} \cdot \mathrm{m}^{2}$.

The controller bandwidth of flux loop is $\omega_{c 1}=200$, and the observer bandwidth is $\omega_{o 1}=2 \omega_{c 1}=400$. The parameters of the flux controller are: $k_{p 1}=200, \beta_{1}=800, \beta_{2}=160000$, $\alpha_{1}=0.5, \delta_{1}=0.01, b_{10}=10$. The controller bandwidth of the speed loop is $\omega_{c 2}=50$, and the observer bandwidth is $\omega_{o 1}=4 \omega_{c 1}=200$. The parameters of the speed controller are: $k_{p 2}=50, \beta_{3}=400, \beta_{4}=40000, \alpha_{2}=0.5, \delta_{2}=0.01, b_{20}=20$. 
B. Disturbance rejection capability of the ESO based controller

In this simulation, the reference values of $\psi_{c r}$ and $n_{r}$ are $0.7 \mathrm{~Wb}$ and $200 \mathrm{r} / \mathrm{min}$, respectively. The BDFM is without load initially, and loaded with $10 \mathrm{Nm}$ at $0.8 \mathrm{~s}$. The PM is powered by $220 \mathrm{~V} / 50 \mathrm{~Hz}$ initially, and the voltage amplitude increases to 240 at $1.2 \mathrm{~s}$. Fig. 7 displays the regulating performances comparison between the proposed controller and a PI controller.

As shown in Fig. 7(a) and (c), there are no overshoots of the flux and rotor speed when the proposed control strategy is utilized, while this is not achieved by the PI controller. The flux and rotor speed track the reference signals quickly and accurately through the proposed controller. As shown in Fig. 7, (b) and (d), the ESOs provide accurate estimations of the disturbances in flux loop and speed loop. There are obvious fluctuations of the flux and rotor speed in the cases of the machine is loaded at $0.8 \mathrm{~s}$ and the power supply changes at $1.2 \mathrm{~s}$. But, thanks to the accurate estimation and compensation for the disturbances via the ESO, this phenomenon does not appear when the proposed controller is utilized. These above results reflect the superiority of the proposed control approach, which has an excellent disturbance rejection capability.
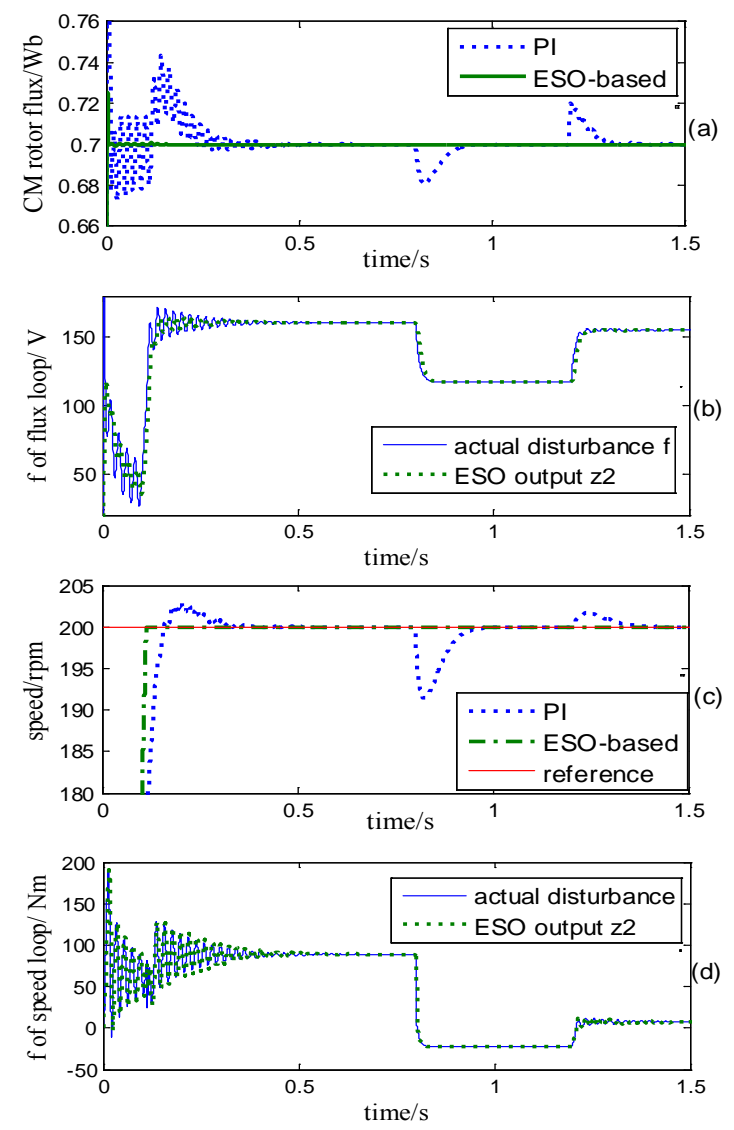

Fig. 7. Disturbance rejection capability comparison between fuzzy ADRC and PI control. (a) CM rotor flux;(b) disturbance in the flux loop; (c) speed; (d) disturbance in the speed loop.

C. Decoupling capability of the ESO based controller As the BDFM is a nonlinear system with strong coupling effects, the flux is strongly coupled with the torque, and also with the rotor speed. This simulation verifies the decoupling capability of the proposed controller. In this simulation, the reference value of the control motor rotor flux amplitude $\psi_{c r}$ is $0.7 \mathrm{~Wb}$ and drops to $0.6 \mathrm{~Wb}$ at $3 \mathrm{~s}$. The reference value of motor speed drops down from $200 \mathrm{r} / \mathrm{min}$ to 0 at $2 \mathrm{~s}$. The BDFM with a load of $10 \mathrm{Nm}$. Fig. 8 shows the control results.

As shown in Fig. 8(e), the electromagnetic torque changes when the reference rotor speed decreases at 2s. The change of torque causes large flux fluctuations in the PI control system. As an improvement, these coupling effects are eliminated by the ESO. So there is no change in the flux when the torque varies in the proposed control system. Similarly, there is a speed fluctuation about $5 \mathrm{r} / \mathrm{min}$ when the flux varies in the PI control system. While this fluctuation disappears when the proposed approach is used. These above results verifies the decoupling capability of the proposed controller.
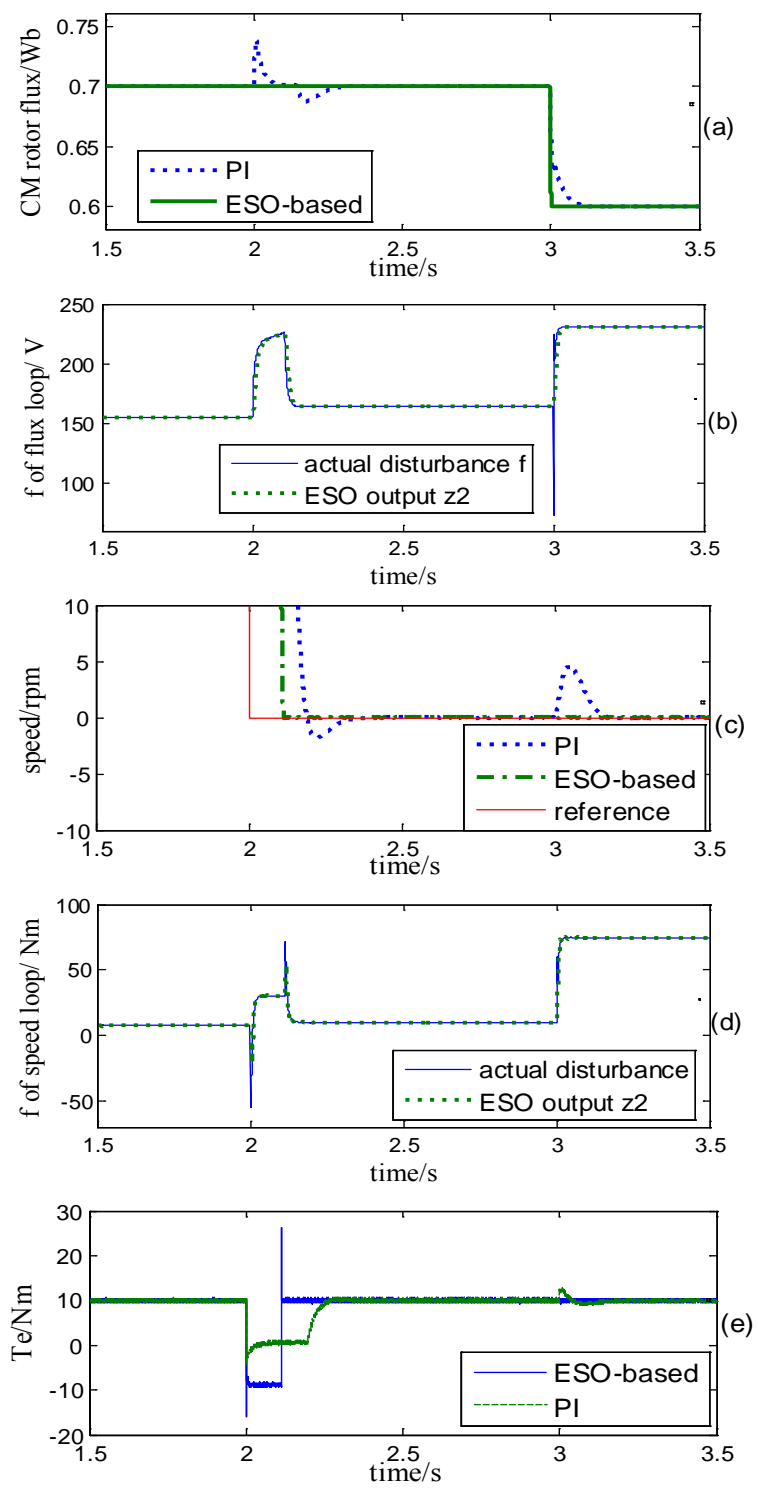

Fig. 8. Decoupling capability comparison between the proposed controller and PI control. (a) CM rotor flux; (b) disturbance in the flux loop; (c) speed; (d) disturbance in the speed loop; (e) electromagnetic torque 


\section{CONCLUSIONS}

An ESO based nonlinear control strategy is presented to address the coupling effects and external disturbances of BDFM in this paper. The ESOs for the flux and the speed are designed to estiminate and compensate the total disturbances. A parameter optimization method is proposed to reduce the workload of parameter tuning based on the parameter configuration rule of a LESO. An excellent observation performance by simpler designing is got.

Simulation results show that the proposed controller achieves fast and accurate regulating without overshoot. The inner coupling effects and the external disturbances, such as the load disturbance and supply disturbance, are eliminated via the ESO exactly. And, the flux and the torque are decoupled. These results verify the disturbance rejection ability and the decoupling ability of the proposed controller.

\section{REFERENCES}

[1] W.K. Song, D.G. Dorrell. "Improved direct torque control method of brushless doubly-fed reluctance machines for wind turbine," IEEE International Symposium on Industrial Electronics (ISIE), pp. 1-5, 2013.
[2] S. Ademi, M.G. Jovanovic. "Vector Control Methods for Brushless Doubly Fed Reluctance Machines,” IEEE Trans. Ind. Electron., vol.62, no.1, pp. 96-104, 2015.

[3] S. Shao, T. Long, E. Abdi, R.A. McMahon. "Dynamic control of the brushless doubly fed induction generator under unbalanced operation," IEEE Trans. Ind. Electron., vol.60, no.6, pp. 2465- 2476, 2013.

[4] A.Gaeta, U. Montanaro. "Application of a robust model reference adaptive control algorithm to a nonlinear automotive actuator," International Journal of Automation and Computing, vol. 11, no. 4, pp. 377-391, 2014.

[5] Z.Q. Gao. "On the foundation of active disturbance rejection control," Control Theory \& Applications, vol. 30, no. 12, pp. 1498-1510, 2013.

[6] Z.Q. Gao. "Scaling and parameterization based controller tuning," In: Proceedings of American Control Conference, Denver, CO, USA, pp. 4989-4996, 2003.

[7] R. Madonski, M. Kordasz, P. Sauer. "Application of a disturbance rejection controller for robotic-enhanced limb rehabilitation trainings," ISA Trans., vol. 53, no. 4, pp. 899-908, 2014.

[8] Q. Zheng, Z.Q. Gao. "Predictive active disturbance rejection control for processes with time delay,” ISA Trans., vol. 53, no. 4, pp. 873-881, 2014.

[9] H. Tang, Y. Li. "Development and active disturbance rejection control of a compliant micro-/nanopositioning piezostage with dual mode," IEEE Trans. Ind. Electron., vol. 61, no. 3, pp. 1475- 1492, 2014. 